Les Cahiers du Centre de Recherches

Archives

18-19 | 1997

Inventaire des archives de Robert Mandrou

\title{
À Nanterre-paris X
}

Jean Lecuir

\section{(2) OpenEdition}

Journals

Édition électronique

URL : http://journals.openedition.org/ccrh/2562

DOI : $10.4000 /$ ccrh. 2562

ISSN : $1760-7906$

Éditeur

Centre de recherches historiques - EHESS

Édition imprimée

Date de publication : 10 octobre 1997

ISSN : 0990-9141

Référence électronique

Jean Lecuir, "À Nanterre-paris X», Les Cahiers du Centre de Recherches Historiques [En ligne], 18-19

1997, mis en ligne le 20 février 2009, consulté le 10 décembre 2020. URL : http://

journals.openedition.org/ccrh/2562; DOI : https://doi.org/10.4000/ccrh.2562

Ce document a été généré automatiquement le 10 décembre 2020.

Article L.111-1 du Code de la propriété intellectuelle. 


\title{
À Nanterre-paris $X$
}

\author{
Jean Lecuir
}

1 À l'occasion de la remise des papiers de Robert Mandrou aux Archives nationales par l'intermédiaire de l'EHESS, d'autres que moi ont exprimé - et fort bien - et leur émotion et leurs souvenirs sur l'enseignant, le chercheur, l'homme, l'ami. Qu'on me permette de développer un aspect généralement peu évoqué de la vie de Robert Mandrou, celui de l'universitaire qui s'est donné, sans compter sa peine, à une nouvelle université de la périphérie parisienne : Paris X-Nanterre. Parce qu'il eût été regrettable de voir dispersée une bibliothèque de spécialiste et que rien ne pouvait mieux témoigner de son attachement à Paris $\mathrm{X}$, Christiane Mandrou lui avait suggéré d'en faire don à cette université... idée qu'il avait immédiatement faite sienne et qui sera concrétisée le moment venu : la fidélité de Christiane Mandrou y veille.

2 Robert Mandrou venait de suivre - et avec quelle attention!- les événements de maijuin 1968 : ceux-ci avaient bousculé sa soutenance de thèse, repoussée en novembre 1968, au moment même où il était élu maître de conférences à Paris $\mathrm{X}$, avant d'être nommé professeur titulaire en histoire moderne en octobre 1970. Nanterre n'était pas pour lui un exil ou une sanction. Il n'était pas de ces universitaires qui ne rêvaient que d'une université intra-muros. Aller à Paris X relevait d'un choix assumé pour son intérêt social, parce qu'il fallait « aller au feu », au devant de vastes auditoires, de ces foules étudiantes passionnées et passionnantes: pour lui, qui avait été secoué par les interpellations étudiantes de l'époque, y compris au sein de son propre séminaire, il s'agissait, en ce lieu universitaire d'où était parti le «mouvement de Mai », de gérer à la fois la haute idée qu'il avait de la fonction de professeur d'université, l'écoute des autres personnels et des étudiants, les attentes positives comme les révoltes et les déceptions post-soixantehuitardes. Il était décidé à apporter pleinement sa pierre à la mutation de l'Université française, et d'abord en se plongeant dans celle de Paris X-Nanterre.

Or les années 1968-1976 furent souvent difficiles, sur un fond d'agitation permanente, scandée par des moments de crise sociale, cours et travaux dirigés interrompus, assemblées générales, manifestations, interventions des forces de l'ordre. Au moindre incident, les heurts violents devenaient réalité. Robert Mandrou était toujours 
rapidement sur les lieux pour s'interposer et essayer de ramener à la raison. Il était de ceux et de celles qui montraient à la fois un grand courage physique et une capacité toujours renouvelée de dialogue avec des étudiants, parfois passablement insupportables. Il défendit ainsi avec constance le devoir de présence des enseignants dans ces moments difficiles, pour aider les autorités universitaires à conserver dignité et autorité à Nanterre et préserver le dialogue avec ces jeunes. Il n'avait pas la mémoire courte en la matière : certains de ses collègues en ont fait parfois la cruelle expérience. Il avait ainsi acquis rapidement une autorité morale sur les étudiants - et pas seulement ceux d'histoire - et sur de nombreux assistants et maîtres assistants du collège $B$.

4 Son action, cependant, visait toujours à ramener chacun dans le cadre institutionnel : Robert Mandrou était profondément un citoyen agissant, un démocrate, vigilant quant au respect de l'état de droit. C'est pourquoi, membre du collège A, il était un assidu des conseils de son UER, veillant à déléguer son pouvoir quand il ne pouvait faire autrement, $\mathrm{y}$ compris à un membre du collège $\mathrm{B}$, et des commissions "pédagogie et recherche ». Il possédait le rare talent de clarifier les problèmes, de forcer chacun à endosser ses responsabilités et de situer les enjeux par la formulation de motions nettes et précises. Toujours concret, il tenait compte des contraintes matérielles et n'hésitait pas à se rallier à un point de vue étudiant ou du collège $\mathrm{B}$, ou à rappeler les difficultés rencontrées par la secrétaire de l'UER, $\mathrm{M}^{\mathrm{me}}$ Bois. Qui voudra bien se pencher sur les comptes rendus officiels de réunion en trouvera de multiples preuves. J'en donnerai trois illustrations. D'abord son intervention le 27 février 1970 :

«L'avenir de Nanterre se joue au niveau de l'enseignement et à celui de la recherche. Sinon, Nanterre sera une faculté de seconde zone, c'est-à-dire que les deux premiers cycles y seraient seuls présents. Les meilleurs seraient alors dirigés sur Paris. Ce serait la sélection. Une université qui ne fait pas de recherche fait de basses besognes.

5 Quelques mois plus tard, le 29 octobre, il demande un bilan public des résultats au contrôle continu et aux examens, par niveau et par sous-section, pour couper court à d'autres rumeurs concernant notre «laxisme » ou notre rigorisme. Le procès-verbal du 5 décembre 1975 cite une lettre qu'il rédigea sur l'indispensable rôle de l'Université en matière de formation scientifique des maitres ${ }^{1}$ et la meilleure façon de le défendre face au ministère, suggérant d'utiliser la presse pour rendre le débat public.

6 Pleinement engagé dans les instances de son université, membre de son conseil scientifique dès 1971, il y a assumé, à partir de cette date, la responsabilité de la commission du budget, qui répartissait chaque année la dotation de recherche de Paris $\mathrm{X}$ entre les quarante-trois centres qui se la disputaient: tâche délicate et pleinement interdisciplinaire. Depuis février-mars 1976, devenu vice-président chargé de la gestion de la recherche, il avait étendu ses responsabilités, auprès du président Verdier, à toutes les rubriques de la vie universitaire où la compétence du conseil scientifique était reconnue. Prenant sa part de la gestion générale de l'université - il était l'un des trois vice-présidents mandatés pour prendre les décisions de maintien de l'ordre en cas de crise -, il évoque ${ }^{2}$ ainsi son rôle :

S'y sont ajoutées, au gré des circonstances et des besoins de l'université, quelques tâches supplémentaires non négligeables; je me contenterai de citer la gestion provisoire de l'UER de sciences juridiques de la mi-septembre jusqu'à la fin de novembre 1976.

7 Face à la cabale politique et à la sécession concertée d'enseignants de cette UER, emmenant leurs postes vers l'université de Paris $\mathrm{V}$, avec la tolérance de la secrétaire 
d'État aux Universités, il avait accepté de Jean-Maurice Verdier la mission d'administrateur provisoire pour essayer de sauver à la fois la dimension pluridisciplinaire de Paris X-Nanterre et l'UER de sciences juridiques : il s'agissait de remobiliser les juristes restés fidèles, de gérer les retombées, en matière d'examen, de la grève printanière de 1976, de faire redémarrer les enseignements et la gestion de l'UER.

Dans une atmosphère conflictuelle, malmené par la presse, notamment d'extrême droite, il ne manqua jamais de faire les mises au point utiles et parvint à rétablir la situation et à préserver la pluridisciplinarité de l'université. Au cours de cette "expérience partie amère, partie vivifiante ", selon ses propres termes, il trouva réconfort dans la pétition des enseignants de Paris $\mathrm{X}$ 《 Quelques vérités sur Nanterre » et dans la mise au point de Jean Imbert, directeur des enseignements supérieurs, à Rivarol. Ce dernier lui écrivit d'ailleurs, le 22 décembre 1976, une lettre manuscrite qui mérite d'être citée en entier : «Cher Monsieur, je vous remercie très vivement de votre lettre du 13 décembre 1976 et du rapport qui y était joint. Ils m'ont confirmé ce que je savais déjà : à savoir que vous avez rempli la tâche qui vous avait été confiée - tâche ô combien délicate ! - avec le sangfroid, la conscience, et le sens des relations publiques que vous avez toujours manifestés. Permettez au directeur des enseignements supérieurs de vous dire sa reconnaissance profonde et de vous féliciter pour l'œuvre d'apaisement que vous avez accomplie. Permettez par ailleurs au collègue - historien du droit - de vous redire qu'il avait confiance dans votre talent, alimenté par une profonde connaissance de l'histoire sociale $»^{3}$.

9 À l'occasion de l'élection de Gérard Couchez comme directeur de l'UER des sciences juridiques, il fut remercié publiquement de son efficacité par le président Verdier qui n'ignorait rien de « la surcharge de travail causée par la gestion de cette monstrueuse UER », ni des « tracas » liés à un engagement total.

10 J'évoquais l'animateur de la recherche et le gestionnaire. Ces fonctions étaient, pour lui, indissociables de l'idée qu'il se faisait de l'Université et de son enseignement. Grâce à ce dépôt d'archives, où l'on trouvera la quasi-totalité de ses cours, il sera possible de montrer l'ampleur des sujets abordés et l'effort de préparation que lui demandait des intitulés toujours ambitieux, qu'ils soient liés à l'agrégation, à la licence - «question générale renouvelée d'année en année »-, passant de l'Allemagne à l'Angleterre, en n'oubliant ni Genève, ni l'Autriche et le monde danubien, ni bien sûr ce qui allait devenir deux livres, les intellectuels des $\mathrm{XVI}^{\mathrm{e}}$ et $\mathrm{xVII}^{\mathrm{e}}$ siècles en Europe ou la raison d'État en Europe. Robert Mandrou était mû par la volonté de donner aux étudiants des repères - clairs et toujours au mieux informés - sur une culture européenne, dont il déplorait les défaillances dans l'Université française. Le Français était en lui indissociable de l'Européen largement ouvert vers l'est du continent, faisant réfléchir ses étudiants sur le rôle des intellectuels dans une société.

11 Mais il innovait aussi dans le cadre du DEUG où, dans la foulée des demandes étudiantes et des réflexions pédagogiques qui suivirent 1968, il inaugura, dès 1970, un enseignement de « méthodologie historique » intégrant, semaine après semaine, des séances de travaux pratiques portant sur des études de textes significatifs. Nous en avions longuement parlé et j'ai eu le plaisir de l'accompagner dans cette démarche novatrice, où l'assistant disposait de la photocopie des notes du cours prononcé et avait pu choisir avec lui des documents adaptés, l'ensemble se voulant au service de la formation méthodologique des étudiants. Vingt-six séances se déroulaient ainsi autour de quatre grands thèmes: histoire et historiographie; la place de l'histoire dans la culture française ; l'histoire, 
science humaine, toute histoire est sociale; histoire, science des hommes agissant, sentant, parlant... Son ambition était de donner ainsi de solides repères sur la démarche scientifique, sur le bon usage du vocabulaire courant, sur les champs de la recherche historique contemporaine. Restait à faire prendre en charge cette innovation, en rotation, par l'ensemble des enseignants, ce qui était une autre histoire.

Et la France, me direz-vous? Robert Mandrou estimait que les étudiants avaient de bons ouvrages de synthèse à leur disposition - il y plaçait à juste raison les siens ; les questions traitées en travaux pratiques permettaient de défricher le vaste chantier français et d'acquérir les bases. Il reprenait en séminaire de maitrise l'information sur les publications récentes et il décortiquait quelque thèse neuve, abordée par toutes sortes d'entrées, obligeant ainsi les étudiants à la découvrir dans le détail. Toujours soucieux de méthode et de précision dans l'expression, agacé par le marxisme de pacotille qui circulait alors, c'est dans ce cadre qu'il avait largement recours à son Classes et luttes de classes au XVII e siècle.

Amoureux de la représentation cartographique et du cinéma, Robert Mandrou soutint aussi l'élargissement des «sciences auxiliaires de l'histoire » au traitement graphique de l'information historique avec S. Bonin, à l'étude de la presse avec M. Martin et aux techniques de l'audiovisuel avec $\mathrm{H}$. Duccini.

Comment ne pas évoquer enfin la manière dont il associait ses collègues assistants et maîtres assistants du collège $B$ à son enseignement spécialisé de troisième année ou à la soutenance des maîtrises étudiantes, toujours à l'écoute de leurs problèmes, s'efforçant de tenir compte des contraintes que chacun avait, d'apporter aide et assistance, dans la discrétion, avec fermeté et bienveillance.

Il en allait de même avec les étudiants, pour lesquels il trouvait toujours le temps d'un rendez-vous, pratiquant le tutorat avant l'heure. Sa vaste culture et sa longue et intime fréquentation des archives lui donnaient une abondante réserve de sujets de recherche, permettant de tenir compte des préférences individuelles. Pourtant, toujours soucieux de son université, il voulait que sa recherche s'ancrât dans son environnement; c'est pourquoi il participa à la constitution de la Société d'histoire de Nanterre, en février 1970, prit contact avec les archives départementales et les municipalités pour connaître les fonds disponibles et susciter, le cas échéant, des travaux.

16 À l'égard des étudiants de maîtrise, il était à la fois exigeant et indulgent, n'oubliant jamais leurs problèmes financiers ${ }^{4}$. Il savait d'expérience qu'il pouvait être difficile de conduire un projet d'études supérieures. Quelques lettres conservées témoignent de ces relations à la fois respectueuses et amicales ; ainsi une étudiante, reçue à l'agrégation, le remercie de l'avoir encouragée à persévérer et s'excuse de ne pas être encore parvenue, malgré son conseil, à suivre le précepte de Lucien Febvre : «Le bon historien aime le vin et le fromage »! À une autre, qui lui faisait part du goût de l'histoire que ses cours avaient accru et de sa volonté d'engager une thèse, il disait : « Prenez votre temps, lisez Michelet, Bloch, Febvre, etc. Jetez un coup d'œil sur des archives départementales en province à l'occasion de vos vacances. "

Robert Mandrou était aussi profondément respectueux du travail de ses étudiants, ne manquant jamais de citer leurs travaux (ses livres en témoignent) ou veillant à la diffusion des meilleures maitrises au travers des microéditions universitaires de la Maison des sciences de l'homme et de l'Association universitaire pour la diffusion internationale de la recherche, chez Hachette ${ }^{5}$. 
Reconnu à Paris X-Nanterre pour ses qualités d'enseignant, de chercheur et d'administrateur, Robert Mandrou aurait pu être candidat à la présidence de cette université, s'il l'avait voulu, en février 1976. Mais, comme il l'a écrit dans une lettre destinée à tout le personnel de l'université le 11 février 1976, il a refusé toute sollicitation en ce sens parce que, dans la conjoncture, il ne s'estimait pas le mieux placé, et pour des « raisons d'abord de santé ». Il se savait, en effet, depuis plusieurs années atteint d'une grave maladie évolutive, sur laquelle il était d'une totale discrétion. L'utilisation de son nom contre Jean-Maurice Verdier, qu'il soutenait - la suite l'a montré -, l'avait contraint à sortir de sa réserve et à faire allusion dans une mise au point publique à des problèmes aussi personnels.

L'année 1977 sera celle des premières conséquences sérieuses du mal. Au point qu'il démissionna en mai 1977 du conseil scientifique et concentra ses forces à l'aboutissement de la Mission historique française auprès de l'Institut Max-Planck de Göttingen, projet auquel il tenait tant. De retour au bout de deux ans, il se mit en congé et prit sa retraite précoce en octobre 1981.

Nous étions nombreux autour de lui et de Christiane Mandrou à l'UER d'histoire pour saluer ce départ inévitable, tous un peu angoissés, connaissant les sautes d'humeur créées par la maladie et le mutisme dans lequel elle l'enfermait. Quand vint, après les discours d'usage, le moment de la réponse de Robert Mandrou, nous vécûmes alors un grand moment, extrêmement émouvant et impressionnant : pendant quelques minutes, il fut à nouveau celui de toujours, disant précisément et gentiment ce que lui avaient apporté cette université, les enseignants qui étaient là, les étudiants, exprimant aussi toute la peine qui était la sienne de partir, la difficulté que représentait ce qu'il devait assumer et l'espoir que ce qu'il avait bâti serait poursuivi. En ce soir de juin 1981, un grand enseignant venait de faire ses adieux à son université.

\section{NOTES}

1. Lors des projets de réforme des programmes d'histoire dans le secondaire, il avait fait voter à l'unanimité une demande de consultation des universitaires. Il avait suscité avec René Rémond, en juin 1973, un colloque sur la place de l'histoire dans les filières nouvelles où quatorze universités étaient représentées ; il était chargé du rapport introductif.

2. Dans un récapitulatif qu'on trouvera dans ses papiers.

3. Le dossier personnel, qu'il avait alors constitué, fut récupéré par mes soins dans un tiroir de son bureau, remis à Christiane Mandrou, et aujourd'hui dans ses archives.

4. Recherches de bourses, par exemple. Il n'acceptait jamais qu'un étudiant paie au restaurant ou au café et adaptait la participation de chacun lors des séminaires provinciaux où il invitait des Nanterrois, utilisant la formule « C'est la part du patron ».

5. Vingt diplômes de maîtrise des années 1970-1973 y trouvèrent place dans la rubrique «histoire des mentalités " dirigée par lui. Il aura suscité de 110 à 120 recherches de maîtrise entre 1969 et 1980, 13 thèses de troisième cycle, 7 thèses d'État. 